\title{
Lactic Acid, DL-
}

National Cancer Institute

\section{Source}

National Cancer Institute. Lactic Acid, DL-. NCI Thesaurus. Code C80130.

The racemic isomer of lactic acid, the biologically active isoform in humans. Lactic acid or lactate is produced during fermentation from pyruvate by lactate dehydrogenase. This reaction, in addition to producing lactic acid, also produces nicotinamide adenine dinucleotide (NAD) that is then used in glycolysis to produce energy source adenosine triphosphate (ATP). 